\section{SAT0556 COST-EFFECTIVENESS OF A BLENDED PHYSIOTHERAPY INTERVENTION IN PATIENTS WITH HIP AND/OR KNEE OSTEOARTHRITIS: A CLUSTER RANDOMISED CONTROLLED TRIAL}

C. Kloek ${ }^{1,2,3}$, J.M. van Dongen ${ }^{4}$, D. Bossen ${ }^{5,6}$, J. Dekker ${ }^{7}$, C. Veenhof $f^{3,8}$ ${ }^{1}$ TRANZO, Tillburg University, Tilburg; ${ }^{2}$ Netherlands Institute for Health Services Research (Nivel); ${ }^{3}$ Research Group Innovation of Mobility Care, HU University of Applied Sciences, Utrecht, ${ }^{4}$ Department of Health Sciences and EMGO+ Institute for Health and Care Research, VU University Medical Center Amsterdam; ${ }^{5}$ ACHIEVE Centre of Expertise, Amsterdam University of Applied Sciences; ${ }^{6}$ Coronel Institute of Occupational Health, Academic Medical Center, University of Amsterdam; ${ }^{7}$ Department of Rehabilitation Medicine and Department of Psychiatry, VU University Medical Center Amsterdam, Amsterdam; ${ }^{8}$ Department of Rehabilitation, Physiotherapy Sciences and Sports, Brain Center Rudolf Magnus, University Medical Center Utrecht, Utrecht, Netherlands

Background: Physiotherapy, consisting of education, graded activity and exercises, is effective in improving levels of physical functioning and pain in patients with osteoarthritis (OA) of hip and/or knee. Blended physiotherapy, in which physiotherapy sessions and an online application are integrated, might support patients in taking an active role in the management of their condition and may reduce disease related costs. Recently, the blended physiotherapy intervention e-Exercise was developed. Bossen 2016 E-Exercise is an integration of five face-to-face physiotherapy sessions with an online application consisting of information-, exerciseand a graded activity module.

Objectives: To evaluate the cost-effectiveness of e-Exercise compared to usual physiotherapy in patients with OA of hip and/or knee, from the societal as well as the healthcare perspective.

Methods: This economic evaluation was conducted alongside a 12 month cluster randomised controlled trial, in which 108 patients received e-Exercise and 99 patients received usual physiotherapy. Clinical outcome measures were qualityadjusted life years (QALYs) according to the EuroQol (EQ-5D-3L), physical functioning (HOOS/KOOS) and physical activity (Actigraph Accelerometer). Costs were measured using self-reported questionnaires. Missing data were multiply imputed and bootstrapping was used to estimate statistical uncertainty.

Results: Total societal costs and total healthcare costs did not significantly differ between groups. Intervention costs $(\Delta \mathrm{C} €-202 ; 95 \% \mathrm{Cl}:-286$ tot -120$)$ and medication costs $(\Delta C €-151 ; 95 \%$ :-340 tot -52$)$ were significantly lower in eExercise compared to usual physiotherapy. No significant differences in effectiveness were found between groups (QALYS $(0-1)$ : $\mathrm{B}=0.01 ; 95 \% \mathrm{Cl}:-0.03$ till 0.04 ; HOOS/KOOS (0-100): $\mathrm{B}=1.49 ; 95 \% \mathrm{Cl}:-4.70$ till 0.69; Accelerometer (min/dag): $\mathrm{B}=-3.46 ; 95 \% \mathrm{Cl}:-11.66$ till 4.73 ). For physical functioning and physical activity, the maximum probability of e-Exercise being cost-effective compared to usual physiotherapy was moderate $(<0.82)$ from both perspectives. For QALYs, the probability of e-Exercise being cost-effective compared to usual physiotherapy was $0.68 / 0.84$ at a willingness to pay of $€ 10.000$,- and $0.70 / 0.80$ at a willingness to pay of $€ 80.000$,- per gained QALY, from respectively the societal and the healthcare perspective.

Conclusions: E-Exercise itself was significantly cheaper compared to usual physiotherapy in patients with hip and/or knee OA, but not cost-effective from the societal- as well as healthcare perspective. The decision between both interventions can be based on the preferences of the patient and the physiotherapist.

\section{REFERENCE:}

[1] Bossen D, Kloek C, Snippe HW, Dekker J, de Bakker D, Veenhof C. A Blended Intervention for Patients With Knee and Hip Osteoarthritis in the Physical Therapy Practice: Development and a Pilot Study. JMIR research protocols 2016;5(1):e32.

Disclosure of Interest: None declared DOI: 10.1136/annrheumdis-2018-eular.3063

\section{SAT0557 \\ 10-YEAR TRAJECTORIES OF PAIN IN EARLY KNEE AND HIP OSTEOARTHRITIS; THE CHECK STUDY}

J. Runhaar ${ }^{1}$, J. Waarsing ${ }^{2}$, D. Schiphof ${ }^{1,1}$, W. van Spil ${ }^{3}$, M. van Middelkoop ${ }^{1}$ S. Bierma-Zeinstra ${ }^{4} .{ }^{1}$ Department of General Practice; ${ }^{2}$ Department of Orthopeadics, ERASMUS MC, Rotterdam; ${ }^{3}$ Department of Rheumatology and Clinical Immunology, UMC Utrecht, Utrecht, ${ }^{4}$ Department of General Practice and Department of Orthopeadics, ERASMUS MC, Rotterdam, Netherlands

Background: Osteoarthritis (OA) is one of the leading causes of chronic pain worldwide. Yet, relatively little is known about the trajectories of pain in early knee and hip OA.

Objectives: To determine subgroups of patients with homogenous patterns of pain over 10 years follow-up, among patients with early hip and/or knee complaints in primary care.
Methods: Data from the CHECK cohort (Cohort Hip and Cohort Knee) were used. For this cohort, 1002 patients between 45 and 65 years at or within 6 months from their first consultation at a general practitioner for symptoms of their hips and or knees, were included and followed for 10 years. A numeric rating scale (NRS) for perceived pain was obtained at baseline and after 2, 5, 8 and 10 years, or until total joint replacement. Using these longitudinal data, subgroups of patients with comparable trajectories over time were identified using Latent Class Growth Analysis (LCGA). Models with 3 to 6 classes when using linear, cubic and quadratic trajectories were evaluated using Mplus software.

Results: At baseline, the 998 subjects ( $79 \%$ women) with $\geq 2$ NRS data points available had a mean age of $55.9 \pm 5.2$ years and a mean BMI of $26.2 \pm 4.0 \mathrm{~kg} / \mathrm{m}^{2}$ In total, 410 patients reported knee pain only (41\%), 173 hip pain only (17\%), and 415 reported both knee and hip pain (42\%) at baseline. On a joint level, 156 knees and 160 hips had $\mathrm{KL}$-grade $\geq 2$ at baseline.

The by LCGA derived models of 3 groups with a linear trajectory and of 6 groups with a cubic trajectory resulted in comparable goodness of fit indicators (Bayesian Information Criteria 17991 vs. 17927, Akaike Information Criteria 17927 vs. 17761 , and entropy 0.694 vs. 0.683 for the 3 and 6 group models, respectively). Both models and the corresponding trajectories are presented in the figure 1. In the 3 group model, the 'high pain trajectory' (group 2, blue line, $n=206$ ) contained most patients with knee and hip complaints (53\%), females (84\%), TJR (21\%) and the highest mean BMI (27.6 \pm 4.6$)$. The 'low pain trajectory' (group 3, green line, $n=441)$ contained most patients with only hip $(19 \%)$ and only knee $(48 \%)$ complaints, and the lowest mean BMI $(25.3 \pm 3.6)$.

In the 6 group model, the 'always high pain trajectory' (group 1, red line, $\mathrm{n}=176$ ) contained most females ( $86 \%)$ and TJR (24\%), and the highest mean BMI $(27.7$ \pm 4.7 ). The 'always low pain trajectory' (group 5, brown line, $n=289$ ) contained most patients with only knee complaints $(51 \%)$ and the lowest mean BMI $(25.0$ \pm 3.4 ). The 'decreasing pain trajectory' (group 2, blue line, $n=37$ ) contained most patients with only hip complaints $(22 \%)$. The 'fluctuating high pain trajectories' (groups $3(n=88)$ and $4(n=142)$, green and pink lines) contained most females $(3: 83 \%$ and $4: 86 \%)$, and patients with knee and hip complaints $(3: 61 \%$ and $4: 44 \%)$.

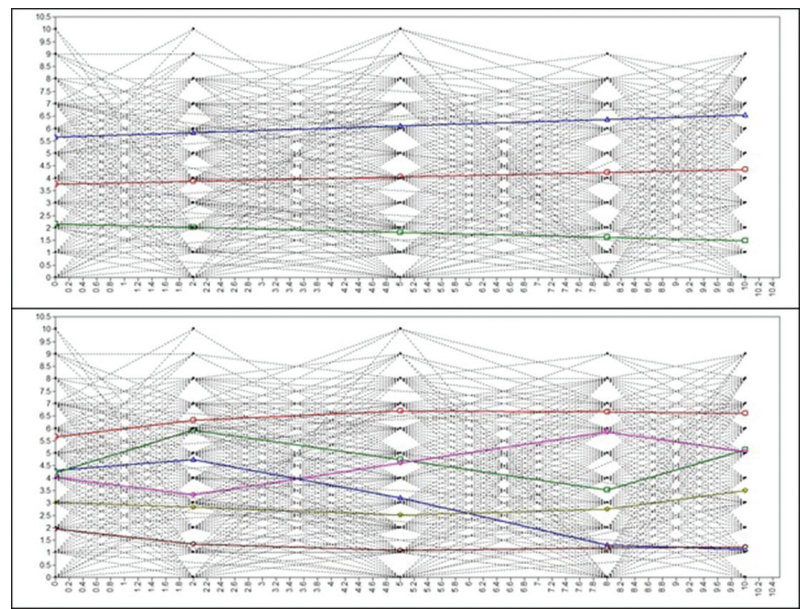

Conclusions: The 6 group model identified more extreme groups with lower minimal and higher maximal prevalence of the presented clinical characteristics. In the end, the conclusion drawn from the two models appear similar; Patients presenting with both knee and hip complaints had less favourable pain trajectories over the following 10 years and the number of total joint replacements was the highest in the groups always reporting high pain scores.

Disclosure of Interest: None declared

DOI: 10.1136/annrheumdis-2018-eular.5267

\section{SAT0558 PHARMACOTHERAPY OF OBESITY IN PATIENTS WITH KNEE OSTEOARTHRITIS AND METABOLIC SYNDROME}

\section{E. Strebkova, L. Alekseeva. Nasonova Research Institute Of Rheumatology,} Moscow, Russian Federation

Background: Osteoarthritis $(O A)$ is one of most common diseases of locomotor system. Different OA phenotypes are currently identified, including metabolic OA associated with meta-inflammation induced by obesity and metabolic syndrome. Objectives: To assess orlistat pharmacotherapy in patients with knee OA (KOA) and metabolic syndrome.

Methods: 50 female patients with Kellgren-Lawrence stage II-III KOA were randomised into 2 equal groups in a 6 months study. All pts were administered lifestyle 